\title{
Trastorno de estrés postraumático en menores internados por maltrato en cinco instituciones ICBF- Pereira
}

\author{
John Jairo Trejos Parra ${ }^{\text {a, b }}$, Claudia Lorena García Osorio ${ }^{\text {a, }}$ \\ ${ }^{a}$ Universidad Tecnológica de Pereira \\ ${ }^{\mathrm{b}}$ Medico, Psicólogo, Magister En Educación y Desarrollo Comunitario, Doctorado en Psicología jtrejos@utp.edu.co \\ ${ }^{\mathrm{c}}$ Profesional en Ciencias del deporte y la recreación, Psicóloga
}

DOI 10.22517/25395203.16531

\section{Resumen}

Introducción. El trastorno de estrés postraumático (TEPT) aparece después de la exposición a un evento amenazante e incluye síntomas de evitación e intrusión del recuerdo, hiperalerta e hiperreactividad, alteraciones negativas cognitivas y alteraciones en el estado de ánimo. Puede aparecer en más del $50 \%$ de los niños afectados por desastres de origen natural o humano, como el maltrato, afectando gravemente su calidad de vida presente y futura.

Objetivo: Determinar la prevalencia de TEPT en niños internados por maltrato en cinco instituciones de Pereira vinculadas al ICBF.

Materiales y métodos. Se construyó, validó y utilizó una escala graduada a partir de los criterios sintomáticos del DSM5 de TEPT adaptada a población escolar. Se aplicó mediante entrevista personal, previa educación lúdica a los niños sobre los conceptos pertinentes.

Resultados y conclusiones. La población de estudio fueron 46 hombres y 47 mujeres institucionalizados en el ICBF, con una media de edad de 11.6 (desviación estándar 2.0) y un tiempo de estadía máximo en el rango inferior de menos de 6 meses con tendencia a la baja y una escolaridad por debajo de lo esperado para la edad. La causa principal de ingreso fue por maltrato intrafamiliar con un $49 \%$, seguido por el abandono o pobreza extrema con un $41 \%$. En cuanto a los criterios para TEPT, el $60(64.5 \%)$ de los menores de edad institucionalizados por vulneración de derechos, cumplían todos los criterios y el $8(8.6 \%)$ cumplían todos menos un criterio sintomático (TEPT subclínico), este alto porcentaje concuerda con la literatura mundial y nacional. Finalmente, el $21.5 \%$ no presentaban síntomas y el $5.4 \%$ no habían estado expuestos a eventos traumáticos. Los diferentes criterios sintomáticos tenían un nivel similar de intensidad.

Palabras clave: Trastorno por estrés postraumático, maltrato infantil.
Post-traumatic stress disorder in abused children that are hospitalized in five ICBF institutions in Pereira.

\begin{abstract}
Introduction. Post-traumatic stress disorder (PTSD) appears after being exposed to a threatening event; it includes symptoms of avoidance and intrusion of memories, an increase of arousal and reactivity, and negative alterations in cognitions and mood. It can appear in more than $50 \%$ of children affected by disasters caused by nature or human beings, such as abuse; these experiences can seriously affect the children's present and future quality of life.
\end{abstract}

Objective. This descriptive research aims to determine the prevalence of PTSD in hospitalized children for mistreatment in five Pereira institutions linked to the ICBF.

Materials and Methods. A graded scale was constructed, validated, and used based on the symptomatic DSM-5 criteria of PTSD and was adapted to the school population. It was applied through a personal interview after giving some playful education about the relevant concepts to the children.

Results and conclusions. The standard population was composed of 46 boys and 47 girls that were part of the ICBF. The main range of age was 11.6 (standard deviation 2.0), the maximum stay time in place was the lowest (less than six months) with a decreasing tendency, and a schooling level below to the expected according to the age. The most common cause of hospitalization was inter-family violence $(49 \%)$ and $41 \%$ for abandonment or extreme poverty. According to the PTSD criteria, 60 youngers $(64.5 \%)$ were hospitalized for violation of rights, and eight children (8.6\%) fit with all but one symptomatic standard (subclinical PTSD). This high percentage tallies with the ones found in the world literature and Colombia. Finally, 21.5\% had no symptoms, and 5.4\% had not been exposed to traumatic events. The different symptomatic criteria had a similar level of intensity.

Key words: post-traumatic stress disorder, child abuse. 


\section{Introducción}

El trastorno de estrés postraumático (TEPT) aparece después de que una persona ha estado expuesta a riesgo de muerte, ha sufrido una lesión grave o un ataque de violencia sexual, ya sea por vivencia directa o por ser testigo del suceso ocurrido a otros. Después del evento traumático, aparecen las siguientes manifestaciones psicológicas: a) evitación del recuerdo de lo ocurrido dado su carácter aversivo; b) intrusión indeseada del recuerdo en la conciencia de la persona, ya sea en estado de vigilia o sueño, causando gran malestar psicológico y fisiológico, llegando en algunos casos a experimentarse como una revivencia del suceso; c) aumento del estado de alerta y de la reactividad en relación con los recuerdos intrusos, estado que afecta el sueño y la capacidad de concentración, lleva a hipervigilancia, exagerada respuesta de sobresalto, irritabilidad $\mathrm{e}$, incluso, a comportamiento imprudente o autodestructivo; d) alteraciones cognitivas persistentes: culpa irracional hacia sí mismo o los demás en relación con las causas o consecuencias del suceso, creencias negativas de sí mismo o de los demás y alteraciones emocionales persistentes: estado emocional negativo con ausencia de emociones positivas y disminución del interés por actividades significativas. Asimismo, se presentan alteraciones disociativas: incapacidad para recordar detalles importantes del suceso y sensación de desapego o extrañamiento de los demás. Todos estos síntomas duran más de un mes y provocan malestar clínico significativo o deterioro de áreas importantes de la vida del individuo $(1)$.

En un estudio de la Organización Mundial de la Salud llevado a cabo en 21 países, se encontró que un 3,6\% de la población mundial había sufrido TEPT en el año 2013 (2) . Más del 10\% de los encuestados declararon haber estado expuestos a eventos traumáticos, ya fuera como testigos $(21,8 \%)$ o por vivencia directa de agresión interpersonal $(18,8 \%)$, accidentes $(17,7 \%)$, conflictos bélicos $(16,2 \%)$ o violencia familiar (12,5\%). En el mundo, entre un $25 \%$ y un $60 \%$ de los adultos refieren haber sufrido un evento traumático en la niñez o la adolescencia, que tuvo un origen humano, por maltrato intrafamiliar o violencia social, o natural, por desastres tipo inundaciones, deslizamientos o terremotos. El TEPT consecuente tiene una prevalencia mundial entre el $6 \%$ y $13 \%$. En el caso de la población infantil, pueden no cumplir todos los criterios para TEP. Sin embargo, su deterioro funcional es similar $_{(3)}$.

En Colombia, según la última Encuesta Nacional de Salud Mental, un $12 \%$ de los escolares (7-11 años) y un $29 \%$ de los adolescentes (12-17 años) ha estado expuesto a evento traumático. De estos, un 39\% y $88 \%$, respectivamente, pueden tener algún problema psicológico postraumático como TEPT (4). A nivel nacional, el Instituto Colombiano de Bienestar Familiar (ICBF) durante el año 2015 recibió un total de 65.472 menores de edad, 1.979 del departamento de Risaralda, que ingresó por vulnerabilidad de sus derechos y adoptabilidad ${ }_{(5)}$. En el mismo año, en el país se atendieron 6.991 por abuso sexual infantil, 161 en Risaralda ${ }_{(6)}$.
El maltrato infantil es "toda forma de perjuicio, castigo, humillación o abuso físico o psicológico, descuido, omisión o trato negligente, malos tratos o explotación sexual, incluidos los actos sexuales abusivos y la violación y en general toda forma de violencia o agresión sobre el niño, la niña o el adolescente por parte de sus padres, representantes legales o cualquier otra persona" ${ }_{(7)}$.

El TEPT generalmente compromete la salud mental de quien lo padece y de las personas que lo rodean, generando un deterioro significativo en su bienestar, proyecto de vida, relaciones interpersonales e inclusión en sociedad ${ }_{(7)}$.En el caso de los niños, los síntomas del TEPT presentan una amplia sintomatología que incluye conductas agresivas, ansiedad, miedos, depresión, problemas de comportamiento, aislamiento, déficit de atención, disociaciones y trastornos del sueño. El TEPT en poblaciones expuestas se eleva al 12\%, y en niños y adolescentes supera el $50 \%$ (8).

Dado lo anterior, se definió como pregunta problema para esta investigación ¿Cuál es la prevalencia del trastorno de estrés postraumático en menores internados por maltrato en cinco instituciones ICBF Pereira 2015?

\section{Metodología}

Dada la importancia de determinar la magnitud de la presencia de TEPT en niños internados por maltrato por el ICBF, se decidió realizar una investigación descriptiva de la prevalencia de este trastorno en cinco hogares vinculados al ICBF de Pereira. Para esto, se solicitó y recibió el visto bueno del "Comité de Bioética" y de la "Vicerrectoría de Investigaciones, Innovación y Extensión" de la Universidad Tecnológica de Pereira.

Primero, se construyó una escala graduada a partir de los criterios sintomáticos del DSM-5 para TEPT, adaptada a la población escolar tanto en el lenguaje verbal como en el lenguaje no verbal, adicionando emoticones y dibujos explicativos. La escala graduada se validó mediante juicio de expertos (tres psicólogos, un psiquiatra, una fonoaudióloga y un licenciado experto en recreación) y mediante una prueba piloto en 39 de estos niños, encontrando un coeficiente alfa de Cronbach de 0.934 (con SPSS 17).

Para la aplicación de este instrumento se realizó una reunión con las directivas de cada institución para presentar la investigación y solicitar la firma del consentimiento informado por el representante legal. Luego, se realizó un encuentro con los niños institucionalizados para explicarles los conceptos de maltrato y estrés postraumático, utilizando videos y dramatizaciones que permitiera que todos los menores lo comprendieran, con el fin de garantizar la calidad de la información por obtener. Después, se diligenció el instrumento mediante entrevista personal. En esta, primero se presentaron y explicaron dibujos sobre los diferentes tipos de maltrato para aclarar y asegurar el entendimiento del concepto por cada niño, posteriormente se le hacía cada pregunta con el apoyo 
de un dibujo y de la explicación del encuestador, finalmente, se le pedía contestar la pregunta en una hoja de respuestas con números y emoticones que indicaban el nivel de cada síntoma.

\section{Resultados y discusión}

\section{Características de la población}

La escala se aplicó a 93 menores de edad internados en cinco instituciones vinculadas al ICBF, 46 hombres y 47 mujeres. Se encontró que el tiempo de estadía en el lugar tenía un descenso logarítmico: menos de 6 meses 55\%, 6-11 meses $21 \%, 12-17$ meses $10 \%, 18-23$ meses $5 \%$, más de 23 meses $2 \%$, este último comprendía a un niño cuya estadía ya era de 30 mese y otro niño con una estadía de 72 meses. La causa principal de ingreso fue maltrato intrafamiliar (físico, verbal social o sexual) con $49 \%$, seguido del abandono o pobreza extrema con el $41 \%$, del fallecimiento de acudientes con $3 \%$ y del desplazamiento forzoso con un 3\%. Finalmente, el $3 \% \sin$ dato específico.

Según el informe del ICBF 2012-2013, tres de cada cuatro casos reportados de maltrato y abuso sexual infantil son perpetrados por sus propios padres o familiares ${ }_{(10)}$. En forma similar, otra investigación en Colombia encontró que: "el 70\% de los incidentes de maltrato ocurrió en el hogar. El abuso sexual fue la primera causa de maltrato infantil, seguido del maltrato emocional, con antecedente de maltrato previo en $54.2 \% "(12)$.

Figura 1. Edades y género de los menores de edad internados en cinco instituciones vinculadas al ICBF. Pereira, 2015.

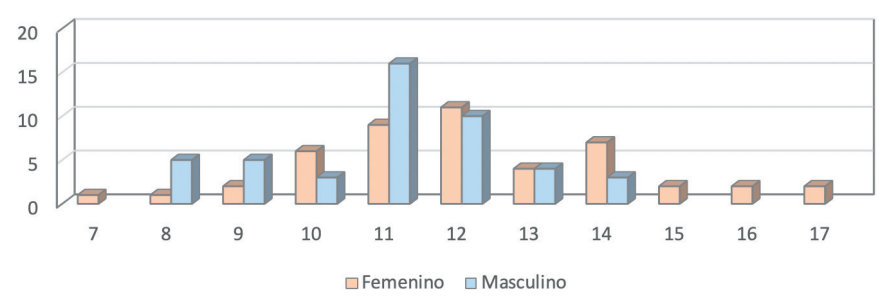

El promedio de la edad fue 11.6, ligeramente mayor en las mujeres (12.1) que en los hombres (11.0), y la desviación estándar fue de 2.0, mayor en las mujeres (2.2) que en los hombres (1.6). En forma similar, en una investigación realizada en Bogotá con 192 casos de maltrato infantil entre 2012 a 2014, se encontró una edad promedio de 8.9 años, $58 \%$ niñas ${ }_{(12)}$. Y es precisamente la edad escolar la más afectada por el TEPT $(14)$.
Figura 2. Escolaridad de menores de edad internados en cinco instituciones vinculadas al ICBF. Pereira, 2015.

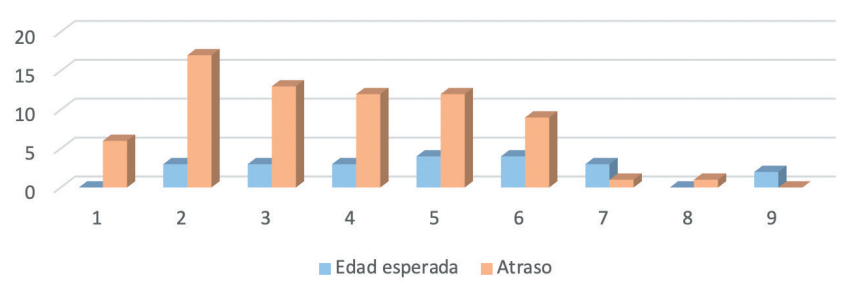

Los niveles de escolaridad están por debajo de lo esperado para la edad. Según la literatura científica, esto es producto de diversos factores, tales como: desinterés por el aprendizaje, generado por la situación de maltrato, insuficiente estimulación, la constante descalificación por parte de los padres que inhibe el desarrollo de la curiosidad y deseo de conocimiento y la ansiedad hacia las relaciones interpersonales por la falta de habilidades en la resolución de conflictos ${ }_{(12)}$

\section{Diagnóstico de TEPT}

Tabla 1. TEPT en menores de edad internados en cinco instituciones vinculadas al ICBF. Pereira, 2015.

\begin{tabular}{|c|c|c|}
\hline Diagnóstico según DSM-5 & $\#$ & $\%$ \\
\hline TEPT & 28 & 30.1 \\
\hline TEPT ¿sin deterioro? & 26 & 28.0 \\
\hline TEPT ¿menor a 1 mes? & 6 & 6,5 \\
\hline TEPT subclínico & 8 & 8,6 \\
\hline Sí exposición no TEPT & 20 & 21,5 \\
\hline No exposición no TEPT & 5 & 5,4 \\
\hline TOTAL & 93 & 100,0 \\
\hline
\end{tabular}

Durante la explicación inicial, al comienzo del diligenciamiento de la escala, se observó que los menores de edad no consideraban que las acciones violentas recibidas por sus padres y familiares eran "maltrato", sino "algo normal". Sólo después de explicarles los tipos de maltrato, comenzaron a identificar que eso les había pasado.

26 internos cumplían con todos los requisitos para TEPT, pero referían que todos estos síntomas no les estaban produciendo malestar significativo o deterioro en su funcionamiento, lo que no es coherente. Tal comportamiento puede deberse a un acostumbramiento a los síntomas, debido a la larga duración del maltrato, de forma que les parecía "normal" sentirse o vivir así; por esto, puede afirmarse que cumplen completamente. 6 menores llenaban todos los requisitos para TEPT, pero recién habían ingresado a la institución, por lo que referían que el último episodio de maltrato había ocurrido hacía menos de 1 mes; sin embargo, como este fenómeno es repetitivo, y no un episodio único, puede considerarse que también cumplían con los criterios. Entonces, se concluye que tenían TEPT 60 (64.5\%) de los menores de edad institucionalizados por vulneración de derechos. Además, 8 (8.6\%) cumplían todos menos un criterio 
sintomático, lo que puede denominarse TEPT subclínico, en coherencia con lo expresado por De la Barra ${ }_{(3)}$. El elevado porcentaje encontrado de TEPT en menores de edad después de desastres (de origen natural o humano) concuerda con la literatura científica mundial, en la cual oscila entre el 30 y el $60 \%{ }_{(14)}$. Igualmente, en Colombia, según la última Encuesta Nacional de Salud Mental, un 39\% de los escolares y un $88 \%$ de los adolescentes expuestos a eventos traumáticos puede estar presentando TEPT $(4)$

El 5.4\% no tenían antecedentes de eventos traumáticos, sino que su ingreso había sido causado por pobreza extrema o muerte de los padres o acudientes. Finalmente, el $21.5 \%$ no presentaban síntomas, aunque sí habían estado expuestos a eventos traumáticos, esto es, eran resilientes. La resiliencia es la capacidad para adaptarse apropiadamente y funcionar con éxito en un entorno perjudicial o de trauma prolongado; en escolares, esto implica un rendimiento académico satisfactorio y competencias psicosociales normales ${ }_{(15)}$.

\section{Criterios sintomáticos de TEPT}

En los 68 menores de edad con TEPT o TEPT subclínico, todos los criterios sintomáticos tienen un nivel similar de intensidad: evitación 53\%, intrusión 49\%, alteraciones negativas $44 \%$ e hiperalerta/hiperreactividad $40 \%$.
El porcentaje en el nivel de los síntomas tiene una media de 45.1 y una desviación estándar de 11.2. Los más sobresalientes $(>50 \%)$ son: evitar recordar (62\%), malestar psicológico (62\%), recuerdos intrusos (59\%), emociones negativas (56\%), sobresalto exagerado (52\%) e irritabilidad (51\%). Se observa que participan síntomas de todos los criterios para TEPT. Los síntomas más bajos están en hiperalerta/hiperreactividad.

\section{Implicaciones psicosociales}

Además de los síntomas propios del TEPT se pueden encontrar: efectos emocionales, como ansiedad, depresión e ideación suicida; auto-percepciones negativas que incluyen baja autoestima, vergüenza y culpa; impactos físicos, en especial déficit en el sistema inmune por el estrés crónico y déficit cognitivos; deterioro en el desarrollo de las habilidades psicosociales, que incluyen pobres habilidades comunicativas, aislamiento y victimización; tendencia a replicar en sus futuros hijos lo vivido en la infancia ${ }_{(16) .}$ Por todo esto, es esencial diagnosticar y manejar oportunamente la situación de maltrato y el TEPT, para disminuir secuelas permanentes ${ }_{(17)}$.

\section{Figura 3. Criterios de TEPT de menores de edad internados en cinco instituciones vinculadas al ICBF. Pereira, 2015.}

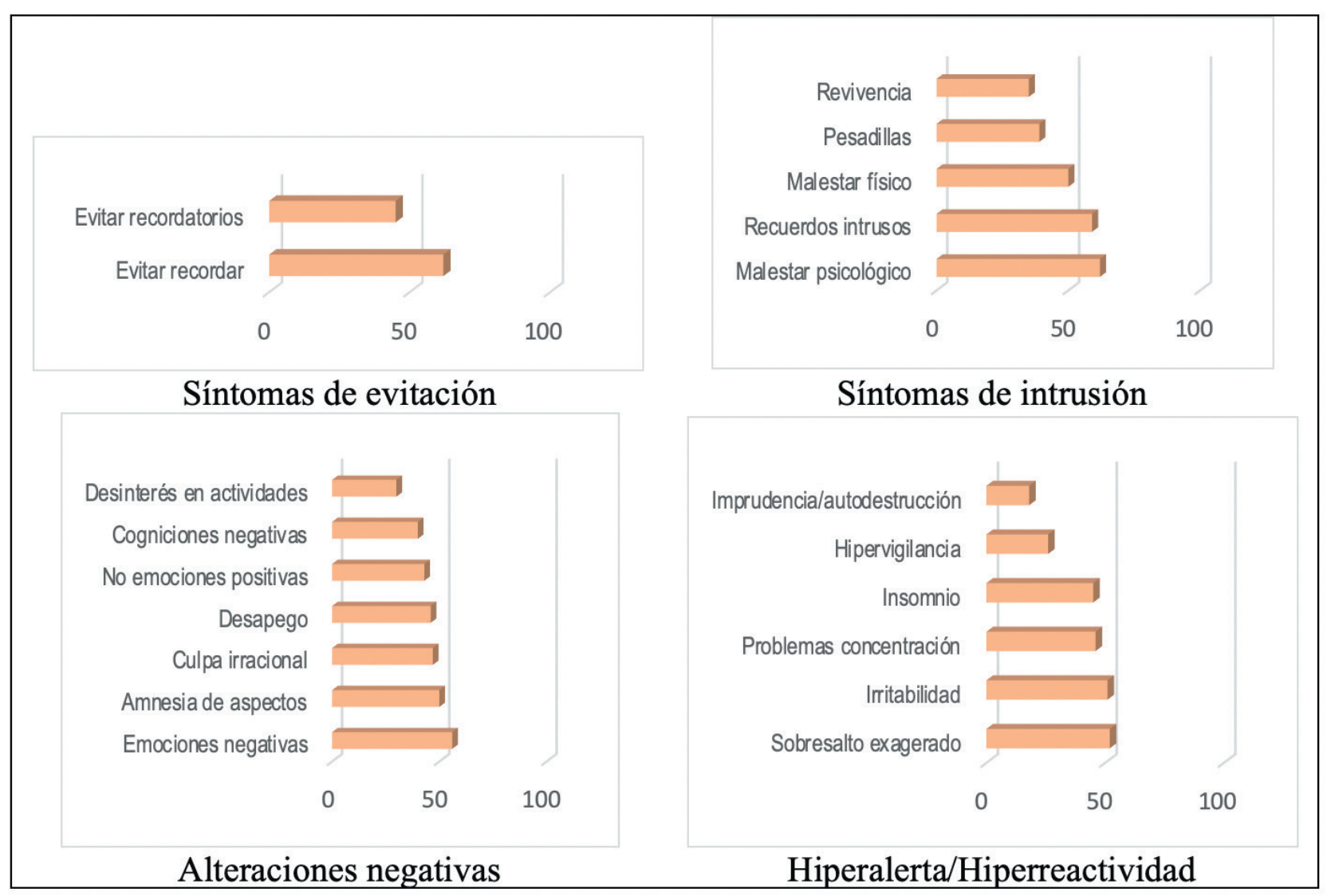




\section{Conclusiones y recomendaciones}

Las características de los 93 menores de edad internados por vulneración de derechos en cinco instituciones vinculadas al ICBF de Pereira eran: igual porcentaje de población masculina y femenina; media de edad de 11.6 (desviación estándar 2.0); tiempo de estadía máximo en el rango inferior de 6 meses; escolaridad por debajo de lo esperado para la edad; causa principal de ingreso por maltrato intrafamiliar 49\%, abandono o pobreza extrema $41 \%$, fallecimiento de acudientes $3 \%$, desplazamiento forzoso 3\%, sin dato 3\%. $60(64.5 \%)$ de los menores de edad institucionalizados por vulneración de derechos cumplían los criterios para TEPT y 8 (8.6\%) cumplían todos menos un criterio sintomático (TEPT subclínico). El $21.5 \%$ eran resilientes, esto es, no presentaban síntomas, aunque sí habían estado expuestos a eventos traumáticos. El $5.4 \%$ no tenían antecedentes de eventos traumáticos, sino que su ingreso había sido causado por pobreza extrema o muerte de los padres o acudientes.

Los diferentes criterios sintomáticos tenían un nivel similar de intensidad. Los síntomas más sobresalientes fueron: evitar recordar (62\%), malestar psicológico $(62 \%)$, recuerdos intrusos $(59 \%)$, emociones negativas $(56 \%)$, sobresalto exagerado $(52 \%)$ e irritabilidad $(51 \%)$, por lo que participan síntomas de todos los criterios para TEPT. Los síntomas más bajos eran hiperalerta/hiperreactividad.

\section{Referencias}

1. Asociación Psiquiátrica Americana. Manual Diagnóstico y Estadístico de los Trastornos Mentales DSM-5 Madrid: Panamericana; 2014.

2. Organización Mundial de la Salud. Word Health Organization. [Online].; 2013. Available from: http://www. who.int/mediacentre/news/releases/2013/trauma_mental_ health_20130806/es/.

3. de la Barra Mac D F. Trastorno de estrés post traumático en niños y adolescentes. Revista chilena de pediatría. 2013; 84(1): p. 7-9.

4. Minsalud, Colciencias y Pontificia Universidad Javeriana. Encuesta Nacional de Salud Mental 2015 Bogotá: Datos, Procesos y Tecnología SAS; 2015.

5. ICBF. Portal Instituto Colombiano de Bienestar Familiar. [Online].; 2016. Available from: http://www.icbf.gov.co/ portal/page/portal/PortalICBF/estadisticas/tablero-proteccion.

6. ICBF. Sistema único de infomación de la niñez del sistema nacional de bienestar familiar. [Online].; 2016. Available from: http://www.suin-snbf.gov.co/suin/Pages/PorIndicador. aspx?id=6.

7. Congreso de la república. Ley 1098 de 2006. Diario Oficial. 2006 noviembre: p. 1-118.
8. Perry BD. Child Trauma Academy. [Online].; 2014. Available from: https://childtrauma.org/wp-content/uploads/2014/10/ PTSD_Spanish_sm.pdf.

9. Mingote JC,MB,II,PA,\&NI. Tratamiento integrado del trastorno de estrés postraumático. Aperturas psicoanalíticas. $2001 ;(8)$.

10. Dirección de Protección ICBF. ICBF. [Online].; 2014 [cited 2015 ene 15. Available from: www. icbf.gov.co/portal/page/portal/Descargas 1/Prensa 1/ ColombiaSinMaltatoInfantil_180313.pdf.

11. Briceño GD, Durán Florez ME, Blanco G, Zorro Cerón S. Epidemiologiade los casoso de maltrato infantil en la organización Sanitas en Bogotá. Revista médica Sanitas. 2015; 18(1): p. 21-28.

12. Peréz-Olmos I, Fernández-Piñeres PE, Rodado-Fuentes S. Prevalencia del Trastorno por Estrés Postraumático por la Guerra, en niños de Cundinamarca, Colombia. Salún Pública. 2005; 7(3): p. 268-280.

13. Salvador Morales L. Los efectos educativos del maltrato infantil. Trabajo de grado. Ciudad del Carmen (México): Universidad Pedagógica Nacional, Licenciatura; 2009.

14. Bados A. Trastorno por estrés postraumático. [Online].; 2015. Available from: http://diposit.ub.edu/dspace/ bitstream/2445/65623/1/TEPT.pdf.

15. Scheeringa. Comentarios al Trastorno por Estrés Postraumático en niños de 6 años y menores. DSM-5. Revista Iberoamericana de Psicotraumatología y Disociación. 2013; $5(1)$.

16. Àlape Zuluaga CJ, Castaño Castrillón JJ, Castaño Quintero LM, Cely Enciso EF, Marín Henao J, Ocampo García DG. Abuso sexual y factores asociados en población escolarizada perteneciente a una institución educativa de la ciudad de Manizales, Caldas (Colombia), 2012. Archivos de medicina. 2013; 13(1): p. 47-60. 